\title{
A survey on matheuristics for routing problems
}

\author{
Claudia Archetti • M. Grazia Speranza
}

Received: 24 July 2013 / Accepted: 5 August 2014 / Published online: 27 August 2014

(C) EURO - The Association of European Operational Research Societies 2014

\begin{abstract}
In this paper, we survey the literature on matheuristics proposed to solve vehicle routing problems. A matheuristic makes use of mathematical programming models in a heuristic framework. Matheuristics have been applied to several different routing problems and include a number of different approaches. We propose a classification in three classes of matheuristics: decomposition approaches, improvement heuristics and branch-and-price/column generation-based approaches. The contribution of this paper is to offer to researchers interested in routing problems a structured overview of the most successful ideas to combine heuristic schemes and mathematical programming models to obtain high quality solutions. Moreover, we analyze the state of the art and provide insights and hints for future research.
\end{abstract}

Keywords Matheuristics - Mixed integer linear programming - Vehicle routing problems · Survey

\section{Mathematics Subject Classification 90C11}

\section{Introduction}

Due to the advances in exact solution methods and hardware technology, several mixed integer linear programming (MILP) models can be solved to optimality or close to optimality within a reasonable amount of time. This has encouraged a number of researchers to design heuristics that incorporate phases where MILP or, more

C. Archetti $(\bowtie) \cdot$ M. G. Speranza

Department of Economics and Management, University of Brescia, Brescia, Italy e-mail: claudia.archetti@unibs.it

M. G. Speranza

e-mail: grazia.speranza@unibs.it 
generally, mathematical programming models are solved, the so-called matheuristics. We will use the definition of matheuristics proposed in Boschetti et al. (2010), i.e., 'matheuristics are heuristic algorithms made by the interoperation of metaheuristics and mathematical programming techniques'. We will consider only approaches embedding the solution of a mathematical programming formulation. The relation between the original problem and the mathematical programming model or models incorporated in a matheuristic may vary significantly.

An early survey on solution approaches combining metaheuristics and exact algorithms was proposed by Puchinger and Raidl (2005). The solution approaches analyzed are both exact and heuristic. Two surveys on matheuristics have been published. The survey due to Ball (2011) covers a large variety of approaches and problems. In Doerner and Schmid (2010), a survey on matheuristics for rich vehicle routing problems is proposed. The scope of this paper is to survey the literature on matheuristics proposed for the solution of all routing problems, with emphasis on the large number of recent contributions. We classify the proposed matheuristics into three classes:

1. Decomposition approaches In general, in a decomposition approach the problem is divided into smaller and simpler subproblems and a specific solution method is applied to each subproblem. In matheuristics, some or all these subproblems are solved through mathematical programming models to optimality or suboptimality.

2. Improvement heuristics. Matheuristics belonging to this class use mathematical programming models to improve a solution found by a different heuristic approach. They are very common as they can be applied whatever heuristic is used to obtain a solution that the mathematical programming model aims at improving.

3. Branch-and-price/column generation-based approaches Branch-and-price algorithms have been widely and successfully used for the solution of routing problems. Such algorithms make use of a set partitioning formulation, where a binary or integer variable is associated with each possible route (column). Due to the exponential number of variables, the solution of the linear relaxation of the formulation is performed through column generation. In the branch-and-price/column generation-based matheuristics the exact method is modified to speed up the convergence, thus loosing the guarantee of optimality. For example, the column generation phase is stopped prematurely.

In the classification used in Ball (2011), besides the decomposition approaches and the improvement heuristics, the class of the approaches using mathematical programming to generate approximate solutions is introduced. Approaches of this class are based on the idea of solving the mathematical programming formulation in a 'relaxed' way, i.e., by relaxing some attributes of the exact solution approach that increase solution time significantly. Methodologies that fall in this class are, for example, the premature stopping of a branch-and-bound algorithm, heuristic variable fixing, and rounding of the relaxed solution. The branch-and-price/column generation-based approaches belong to this class and are the only approaches of this class used for the solution of vehicle routing problems. In Ball (2011) a specific section is devoted to the relaxation-based approaches which is a subset of the previous one, due to the numerous contributions. These approaches are based on the solution of a relaxation of the mathematical programming formulation and on the subsequent construction of a feasible solution on 
the basis of the information provided by the relaxation. Few contributions belonging to this latter class have been proposed for routing problems and we included each of them in one of the three classes we consider.

The classification proposed in Doerner and Schmid (2010) is different from the one adopted in Ball (2011). The following classes are introduced:

1. set-covering/partitioning-based approaches, corresponding to the class of branchand-price/column generation-based approaches;

2. local branching approaches, based on the local branching scheme proposed in Fischetti and Lodi (2003);

3. decomposition approaches, which coincides with the first class defined in Ball (2011).

We decided not to adopt this classification since very few algorithms for routing problems fall into the second class. Although the survey by Doerner and Schmid (2010) is quite recent, several papers appeared in the most recent years and are not covered. In addition, some classes of routing problems are not covered.

The following three sections are devoted to the description of the three classes of approaches, namely the "Decomposition approaches", the "Improvement heuristics" and the "Branch-and-price/column generation-based approaches". In "Summary and conclusions" we provide a summary of the survey, reporting in a table an overview of the approaches surveyed, and draw some conclusions.

\section{Decomposition approaches}

Decomposition approaches are based on the idea of identifying subproblems which are smaller and easier to solve than the original problem. The subproblems are handled and solved independently. Finally, a feasible solution for the original problem is obtained from the solutions to the subproblems. In matheuristics, one or all the subproblems are solved through the exact solution of a mathematical programming formulation. Sometimes a time limit is imposed to control the computational time in case of large size instances, thus possibly loosing the optimality of the solution found. As in all contributions that we review the mathematical programming model is a MILP model, for the sake of brevity, we will refer to MILP models in the following.

Due to their nature, the decomposition approaches are particularly suitable for the solution of complex and integrated problems. This is the case for the vehicle routing problems (VRPs), the inventory routing problems (IRPs), the production routing problems (PRPs) and the location routing problems (LRPs). Different matheuristics have been proposed for the solution of these problems belonging to the class of decomposition approaches.

Routing problems typically involve the following two basic decisions (in addition to further decisions related to the particular application): the clustering of customers which are assigned to each vehicle and the sequencing of customers in vehicle routes. This feature makes it natural the use of a decomposition approach of the kind cluster first-route second, i.e., an approach where first the assignment of customers to vehicles is made and then the decision on how to route the customers assigned to each vehicle is taken. 
In the following, we classify the decomposition approaches on the basis of the kind of approach proposed. We identified the following classes: cluster first-route second, two-phase approaches, partial optimization approaches and rolling horizon approaches. Before describing these approaches in details, we mention that the cluster first-route second approaches can be seen as a subclass of the two-phase approaches. However, we decided to dedicate a specific class to them as, due to the nature of routing problems, most of the approaches belong to this class.

\section{Cluster first-route second approaches}

The basic idea of the cluster first-route second approaches is to divide the two main decisions that characterize routing problems, i.e., the assignment of customers to vehicles and the sequencing of the customers visited by each route.

Cluster first-route second is one of the first heuristic approaches proposed for the solution of the classical VRP, that for the sake of brevity we call VRP. In the VRP we are given a set of customers with an associated demand and a fleet of capacitated vehicles. The problem is to find a set of vehicle routes serving these customers such that the demands are satisfied, each customer is visited only once and the vehicle capacity is never exceeded. For a survey on properties, solution approaches and variants of the VRP the reader is referred to Toth and Vigo (2002) and Golden et al. (2008). A matheuristic based on a cluster first-route second approach to solve the VRP is motivated by the fact that the clustering of customers can be handled through the solution of a MILP. The routing of customers inside each route can instead be managed by adopting any heuristic available for the solution of the Traveling Salesman Problem (TSP).

The first authors who proposed a cluster first-route second matheuristic for a routing problem, and specifically for the VRP, are Fisher and Jaikumar (1981). In the first phase of the algorithm so-called seed customers are chosen heuristically, and an assignment problem is solved to optimality to assign the remaining customers to the seed customers. Each seed customer identifies a cluster of customers. Then, routes are built by solving a TSP on each cluster. This approach can be applied to a wide variety of routing problems. The scheme has later been extended to solve the VRPTW in Koskosidis et al. (1992). Bramel and Simchi-Levi (1995) propose a decomposition approach for the VRP which is similar to the one proposed in Fisher and Jaikumar (1981). The algorithm is based on the formulation of the routing problem as a capacitated concentrator location problem (CCLP). The idea is to identify seed points, to estimate the cost of assigning each customer to each seed point and then solve a CCLP to determine the clustering of customers. Once the clusters are obtained, a TSP is solved on each cluster. The authors apply the algorithm to the VRP (and to an IRP) showing that the heuristic performs well on both problems and often outperforms previous heuristics proposed in the literature. A similar approach is applied by the same authors to the VRPTW in Bramel and Simchi-Levi (1996).

The IRPs combine the construction of delivery routes with the management of inventory at customers and supplier. Typically, a single supplier is considered. Goods have to be distributed from the supplier to a set of geographically dispersed customers 
over a specified planning horizon. Customer demands have to be satisfied by capacitated vehicles and the objective is to minimize the total cost given by the sum of the routing and inventory costs. A variety of IRPs have been studied in the literature. The reader is referred to Bertazzi et al. (2008), Campbell et al. (1998), Cordeau et al. (2009) and Federgruen and Simchi-Levi (1995) for surveys on the subject. In Bertazzi and Speranza (2012) different ideas for matheuristics for IRPs are presented. The PRPs are extensions of the IRPs which include production decisions at the supplier (also called production plant in the PRPs). Production cost is typically taken into account in the objective function. In Chandra and Fisher (1994), a matheuristic for the PRP is proposed where the production schedule is determined in the first phase together with delivery quantities. A TSP heuristic is then used in the second phase to build the delivery routes.

The approaches previously mentioned are 'one-shot' approaches, i.e., once the two phases are completed the algorithm terminates. More advanced approaches have embedded the cluster first-route second idea in an iterative scheme. We now describe these contributions.

One of the first contributions on iterated cluster first-route second approaches is due to Federgruen and Zipkin (1984) for the solution of an IRP. The authors consider a problem where customers have a stochastic demand and where backlogging is allowed, but generates a penalty cost. Their solution procedure is composed of two phases. In the first phase, the inventory allocation problem is solved to optimality by means of a MILP. In the second phase, vehicle routes are built using classical VRP heuristics [like the $r$-opt heuristic by Lin and Kernighan (1973)] which have been adapted to take into account inventory allocation. The solution obtained in the second phase is used to modify the objective function of the MILP solved in the first phase, and the procedure is iterated. Campbell and Savelsbergh (2004) consider an IRP with a long time horizon and no inventory costs. Given the long time horizon, the solution approach decomposes the problem in two hierarchical phases. In the first phase, the decision process is focused on assigning customer deliveries to days. In the second phase, the horizon is shortened and vehicle routes are constructed. The solution obtained in the second phase is used to update the input of the first phase. The procedure is repeated on a rolling-horizon base.

In Absi et al. (2014), the authors study a PRP where inventory costs are taken into account both at the production plant and at the customers, while production induces fixed and variable costs. The authors propose an approach which is similar to the one proposed in Chandra and Fisher (1994). The approach, in a first phase, solves a MILP model to determine the production and delivery quantities. A heuristic is then used to create the vehicle routes for each period. In the first phase, an approximation of the routing cost is used in the objective function. The solution obtained in the second phase is used to update the routing cost in the first phase, and the procedure is repeated iteratively.

In Prins et al. (2007) the capacitated LRP is studied. The LRPs are problems where one has to decide at the same time the location of supplying facilities and the distribution of goods from these facilities to a set of customers. Thus, the LRPs add to the VRPs the location decisions making the problems remarkably harder to solve. This is the reason why the literature has focused on heuristic algorithms [see Min et al. 
(1998)]. In the capacitated LRP, a capacity constraint is imposed on each supplying facility. In Prins et al. (2007) a decomposition approach is proposed where, in the first phase, location decisions are taken by solving a facility location problem through a Lagrangian relaxation approach. Then, routes are built in the second phase through a granular Tabu Search (TS) heuristic. The procedure is repeated iteratively.

In Guerrero et al. (2013), a cluster first-route second matheuristic is proposed for an inventory location routing problem (ILRP) which is an IRP with multiple suppliers where, in addition to establishing the distribution plan and the vehicle routes, the locations of supplier plants have to be determined. The objective is to minimize the sum of location costs, inventory costs and transportation costs. The authors present a matheuristic where, in the first phase, a MILP model is solved to determine the locations of the supplier plants and the distribution plan. Vehicle routes are constructed in the second phase through a local search and an intensification procedure.

\section{Two-phase approaches}

We insert in the class of two-phase approaches the algorithms that decompose the problem in two phases and solve them separately, but do not belong to the cluster first-route second class. There are many different ways of decomposing problems. With the exception of the cluster first-route second principle which is the most widely used in routing problems and the most successful one, other criteria proposed in the literature are based on the specific nature and structure of the problem at hand.

An example of a two-phase matheuristic is the one proposed in Yi and Özdamar (2007) where a logistic problem arising in disaster response activities is studied which can be modeled as an LRP. The problem is complicated by the presence of many side constraints. The authors propose a mixed integer multi-commodity network flow model that treats vehicles as integer commodity flows. The heuristic solution approach is structured in two phases. In the first phase, a simplified version of the mathematical formulation is solved in order to obtain location decisions and an approximation of vehicle routes. In the second phase, the information gathered from the first phase is used to build detailed vehicle routes and to assign a schedule to each vehicle. A different two-phase approach is developed in Yu et al. (2008) for the IRP with split deliveries. The authors propose an approximation model which is solved through a Lagrangian relaxation method. The relaxed problem is decomposed into an inventory problem solved through linear programming and a routing problem solved through a minimum cost flow algorithm. The solution of the model is used to construct a feasible IRP solution by solving a series of assignment problems.

Flisberga et al. (2009) study the problem of determining the daily routes of logging trucks in forestry. This is a pickup and delivery problem complicated by several side constraints. The authors propose a two-phase approach where, in the first phase, they solve a linear programming (LP) model to find the flow from supply points to demand points. Then, demand and supply points are coupled and represented as single nodes. The resulting problem is a VRPTW which is solved through a TS. A different logistic problem is the one analyzed in Halvorsen-Weare and Fagerholt (2013), which is a routing and scheduling problem arising in maritime logistics. The solution 
methodology divides the routing decisions from the scheduling decisions. The routing decisions involve the assignment of cargo to vessels and the routing of each vessel while the scheduling decisions establish the service time of each cargo. The routing subproblem is solved through a heuristic based on inter-route local search operators. The scheduling subproblem is solved through the exact solution of a MILP model. Hemmelmayr et al. (2014) study instead a routing problem arising in the design of a waste collection system. The problem is to determine the size of the bins located on the streets and to plan the vehicle routes. The authors propose different solution approaches where the routing part is solved through a variable neighborhood search (VNS) heuristic while the problem of determining the best size of the bins is solved through a MILP model. Two hierarchical approaches are proposed: one where the bin allocation is determined first and the routing then, and one where the routing is planned first and the bin allocation then. Moreover, they propose two integrated approaches where the bin allocation problem is solved at each iteration of the VNS.

Two bus routing problems are studied in Schittekat et al. (2013) and Walteros et al. (2014). Schittekat et al. (2013) study the school bus routing problem which is a routing problem where three simultaneous decisions have to be taken: determine the set of stops to visit, define for each student which stop he/she should walk to, and generate vehicle routes that minimize the distance traveled. They propose a heuristic approach where the routing part is solved through a greedy randomized local search, to get an initial solution, and a variable neighborhood descent (VND). Once the routes are given, a MILP model is solved to assign students to stops. Walteros et al. (2014) study the bus rapid transit route design problem that is the problem of finding a set of routes and frequencies that minimize the operational and passenger costs in a bus rapid transit system. The authors propose a hybrid genetic algorithm where the evaluation of the fitness of each solution is made through the optimal solution of an LP program. The LP is solved through column generation.

Finally, a home health care problem is studied in Allaoua et al. (2013) which is the problem of determining optimal routes and rosters for the health care staffs. A twophase approach is proposed where the rostering part is solved through a set partitioning formulation while the routing part is handled by solving to optimality a Multi-depot TSP with Time Windows.

\section{Partial optimization approaches}

The idea of partial optimization approaches is that one or more MILP models are used to handle a part of the problem while keeping constant all decisions related to the remaining parts. Typically, the MILP models are used to handle the part which does not include the routing decisions, which are the most difficult to handle through a mathematical formulation.

In Coelho et al. (2011) an IRP with transshipment is studied. The authors propose an optimization-based adaptive large neighborhood search (ALNS). Several heuristics are used to search the solution neighborhood. Every time a heuristic operator is applied to the current solution, a MILP model is solved to determine the best value of the delivery quantities to each customer. The methodology is applied by the same authors 
in Coelho et al. (2012) to solve an IRP with multiple vehicles and different consistency requirements, i.e., quantity consistency, vehicle fulfilling rate, order-up-to level policy, driver consistency, partial driver consistency and visit spacing. A similar methodology is applied in Adulyasak et al. (2012) for the solution of a PRP. Heuristic operators are used to determine the delivery days and vehicle routes, while a network flow model is optimally solved to determine production and delivery quantities.

Demir et al. (2012) propose an ALNS for the solution of the pollution routing problem (PLR), an extension of the VRPTW where the speed on each route has to be determined in order to minimize an objective function comprising fuel, emission and driver costs. Classical heuristic operators are used to search the solution space and a MILP model is solved to determine the best speed for each route segment.

Goel and Meisel (2013) analyze the problem of routing and scheduling workers for the maintenance of an electricity network. The objective is to assign each maintenance task to a worker and to determine a schedule such that the downtimes of power lines and the travel effort of workers are minimized. A large neighborhood search (LNS) approach is proposed where the routes are constructed through heuristic methods and the information given by the solution of the relaxation of the problem formulation is used. Once the routes are fixed, the scheduling part, that is the problem of determining the starting time of each task, is solved through the optimal solution of a LP model.

\section{Rolling horizon approaches}

Rolling horizon heuristics (RHH) are solution methodologies applied to problems where decisions must be taken over time. The idea is to consider a short time period and solve the corresponding subproblem. The decision taken by the solution of a subproblem is used to update the information for the following subproblem.

This methodology was embedded in a matheuristic framework in Rakke et al. (2011), where an IRP arising in maritime logistics is studied. The authors propose a RHH where the planning period is partitioned into short intervals. Moreover, each interval is divided in a frozen period, a central period and a forecast period. A MILP model is solved for each interval where the values of the variables in the frozen period are inherited from the values of the variables of the central period in the previous interval, integrality is retained for the variables of the central period while integrality is relaxed for the forecast period.

In Agra et al. (2014), the authors propose a matheuristic which is a combination of different approaches presented earlier for the solution of an IRP arising in maritime logistics. In particular, they propose a mathematical programming formulation of the problem and a heuristic which uses a rolling horizon decomposition, local branching and a feasibility pump procedure based on the iterative rounding of fractional variables.

\section{Remarks}

Decomposition approaches are based on the idea of generating subproblems which are simpler than the original problem at hand and whose solutions can be combined to obtain a feasible solution for the original problem. The success of a decomposition 
approach highly depends on the ability to find the right subproblems and to properly integrate their solutions. A matheuristic based on decomposition solves some of the subproblems through MILP models. For the procedure to be successful, it is crucial to either define a 'one-shot' procedure where each subproblem preserves the main characteristics of the original problem and to obtain a good solution from the subproblems, or to develop an iterated procedure where, at each iteration, information is exchanged among subproblems in order to improve the solution quality.

\section{Improvement heuristics}

Matheuristics belonging to the class of improvement heuristics combine a heuristic with the exact solution of a MILP model that aims at improving the solution obtained by applying the heuristic. Different ways to combine the heuristic procedure and the solution of a MILP model have been developed. The MILP model may be solved after the heuristic and applied to the solution obtained by the heuristic in order to improve it. In other cases, the MILP model is used to generate an initial solution. Finally, the MILP model may be integrated into the heuristic phase to improve the search process. In the following, we classify the improvement heuristics on the basis of the kind of approach proposed. More specifically, the approaches are distinguished on the basis of the fact that the MILP models are solved only once, and in this case we call them one-shot approaches, or that MILP models are used for local optimization.

\section{One-shot approaches}

One-shot approaches are heuristic schemes where a MILP model is solved once in order to improve a feasible solution found by a heuristic. Typically, the solution of the MILP model is performed at the end of the algorithm to improve the best solution found. We have included in this class also approaches where different MILP models are solved. The MILP models are solved only once, contrary to what happens in the approaches where MILP models are used for local optimization, described in the following section.

An example of one-shot approaches is the one proposed by Savelsbergh and Song (2008) for the so-called IRP with continuous moves, which is an IRP where many supplying facilities are given and vehicles start a trip from a facility and may end it at a different one, from where a new route is started. The authors propose an integer multicommodity flow formulation to model the problem based on a time-expanded graph. The formulation is applied to improve a feasible solution found by a greedy randomized heuristic. A similar idea is used in Song and Furman (2013) for the solution of an IRP arising in maritime logistics. In Stalhåne et al. (2012) the same problem studied in Rakke et al. (2011), namely an IRP arising in maritime logistics, is considered. A matheuristic is proposed that is a construction and improvement heuristic where a multi-start local search heuristic constructs a set of solutions using a greedy insertion procedure. The solutions are then improved in the intensification phase by applying a branch-and-bound algorithm on a MILP model where a subset of variables of the original problem formulation are fixed to a given value. 
A further example of one-shot approaches is the one proposed in Archetti et al. (2014c) for the solution of the directed profitable rural postman problem (DPRPP). The DPRPP is a generalization of the directed Rural Postman Problem where a profit is associated with a subset of the arcs of the graph. The objective of the DPRPP is to find a tour that maximizes the difference between the total collected profit and the traveling cost. The solution approach proposed in Archetti et al. (2014c) is based on a TS algorithm which repeatedly removes and inserts customers in the tour. Intensification and diversification phases are also applied to improve solutions. The TS terminates when a certain number of iterations have elapsed without improving the incumbent solution. At this point, the set of profitable arcs is partitioned in three subsets: good, bad and dubious arcs. This partition is based on the number of times each arc has been traversed in a solution of the TS. Bad arcs are discarded while a tour is found on the good arcs. Then, a MILP model is solved to optimally insert dubious arcs in this tour.

The free newspaper delivery problem (FNDP) is the problem of determining the distribution of free newspapers from the production plant to a number of subway and tram stations dispersed in the urban area. In Archetti et al. (2013d), a matheuristic is proposed where the problem is decomposed in two subproblems: the creation of a delivery plan and the routing. The creation of a delivery plan is made by solving to optimality a MILP model. For the routing phase, a simple insertion heuristic is applied and eventually improved through an LNS. When the routing subproblem is solved, a feasible solution is obtained. Then, a MILP model is solved which tries to reduce the number of trips by changing the delivery quantities determined in the first subproblem. This algorithm is an example of one-shot approach where two MILP models are solved, one at the beginning of the solution procedure, to construct a starting delivery plan, and one at the end to improve the solution found. Note that this algorithm combines a two-phase approach, as it decomposes the problem in two phases, with an improvement heuristic, as the final MILP aims at improving the best solution found at the end of the previous two phases.

Approaches with MILP models for local optimization

The basic idea of this class of approaches is to use MILP models as tools to explore the solution space. Thus, they are used as operators inside a searching procedure. They can be used in different ways: as a tool to explore the neighborhood, as intensification tools or as an operator to complete a partial solution. Most of the approaches belonging to this class presented in the literature for the solution of routing problems use MILP models as intensification tools.

$\mathrm{Xu}$ and Kelly (1996) propose an iterated approach where a MILP model is used to explore the neighborhood. The algorithm is a TS heuristic based on a network flow model which simultaneously evaluates several customer ejection and insertion moves. Heuristic procedures for the TSP are applied to improve vehicle routes. Another approach where the MILP model is used to explore the neighborhood is proposed in Franceschi et al. (2006) where an initial solution is constructed by taking the best solution known in the literature. The procedure then removes chains of customers from the solution. A number of insertion points are determined in the partial solution 
and the removed customers are organized in a large number of chains. Then a MILP model is solved to optimally insert the removed customers in the insertion points. The procedure is repeated iteratively. A similar approach is proposed in Salari et al. (2010) to solve the open VRP that is a VRP where vehicles do not have to return to the depot. Further examples where a MILP model is used to explore the neighborhood are those proposed in Rei et al. (2010) and Maraš et al. (2013) which both apply a heuristic local branching where, at each iteration, a constraint is added to the MILP formulation in order to find a new solution with a Hamming distance with respect to the current solution not greater than a given threshold.

Approaches where the MILP models are used as intensification tools are more numerous and analyzed in the following. The idea is similar to the one used in the approaches described above, i.e., MILP models are again used to explore the neighborhood. The difference with respect to the previous approaches is that the MILP models are used as intensification tools, and are solved only at certain steps of the solution algorithm and not at every iteration.

In Yildirim and Çatay (2014), a set covering formulation is used to choose the best routes generated by an ant colony optimization (ACO) algorithm for the solution of the VRP. The solution obtained through the MILP model is then used by the ACO to update the pheromone trails. The procedure is repeated iteratively.

In Schmid et al. (2010), a rich VRP for the delivery of ready-mixed concrete is studied. The authors propose a VNS and a very large neighborhood search (VLNS) where a MILP model is solved at each iteration which improves the current solution. To reduce the computational time, some variables are fixed in the MILP model. The number and the set of variables to be fixed are determined by the neighborhood definition.

Chen et al. (2007) propose the first matheuristic for a split delivery vehicle routing problem (SDVRP). The SDVRPs are variants of the classical VRPs where each customer is allowed to be visited more than once, if beneficial, even when its demand is smaller than the vehicle capacity. The interest in studying the SDVRP is growing in the recent years due to the savings that can be achieved by allowing split deliveries. For surveys on the SDVRP, the reader is referred to Archetti and Speranza (2008, 2012). The idea of the approach proposed in Chen et al. (2007) is the following: an initial solution is obtained by applying the classical Clarke and Wright algorithm for the VRP. This solution is then improved by solving a MILP model which reallocates the endpoints of each route in order to decrease the traveling cost. Finally, the solution is post-optimized using the variable length record-to-record travel algorithm developed in Li et al. (2005) for the VRP. The procedure is iterated for a certain number of iterations. A similar procedure is applied in Gulczynski et al. (2010) to the SDVRP with minimum delivery amounts, that is an SDVRP where each delivery to a customer should exceed a minimum amount.

A different approach is proposed to solve an IRP on a discretized time horizon in Archetti et al. (2012). The algorithm is a TS combined with an intensification technique which consists in solving a sequence of MILP models. This intensification is applied every time the incumbent solution is improved with the aim to improve it further. A similar idea is applied in Archetti et al. (2013c) for the solution of an arc routing problem with profits, the team orienteering arc routing problem (TOARP). The TOARP 
is the arc routing counterpart of the more famous team orienteering problem (TOP). In the TOP, customers correspond to the nodes of the graph and a profit is associated with each customer. A limited fleet of vehicles is available and the objective is to find vehicle routes such that the total collected profit is maximized and the duration of each route does not exceed a given threshold. In the TOARP customers correspond to arcs. The problem setting remains the same as in the TOP. A matheuristic is proposed in Archetti et al. (2013c) which is based on a VNS scheme. The VNS repeatedly removes and inserts customers into the solution. Every time a new best solution is found, each route is improved by solving to optimality an asymmetric TSP on a transformed graph where vertices correspond to profitable arcs served in the current solution. Moreover, before changing the neighborhood size, two MILP models are solved to improve the incumbent solution. The first is a set partitioning model which chooses the best routes among all routes generated so far by the VNS. The second MILP model modifies the current solution inserting and removing chains of customers.

Ngueveu et al. (2010) propose an approach which differs from the ones described above as it uses a MILP model to reduce the size of the neighborhood evaluated at each iteration. The authors study the $m$-peripathetic VRP, namely the problem of finding a minimum cost set of routes over $m$ periods such that each customer is visited exactly once, no edge is traversed more than once and vehicle capacity is never violated. The authors propose a TS heuristic where moves are defined by exchanges of edges. In order to reduce the neighborhood size, promising edges are identified by solving a $b$-matching problem. As promising edges are likely to be part of an optimal solution, they are used to restrict the neighborhood size. Another approach where a MILP model is used to reduce the neighborhood size is the one presented in Wolfler Calvo and Touati-Moungla (2011) for the solution of a dial-a-ride problem. The algorithm is a granular TS where the granularity of the solution space is determined through the solution of an assignment problem which identifies most promising edges. Then, the solution space is searched by using an edge ordering based on the information given by the solution of the assignment problem.

An example of matheuristic where the solution of a MILP model is used as an operator to complete a partial solution is presented in Rodríguez-Martín and SalazarGonzález (2012) for the solution of the multi-commodity one-to-one pickup-anddelivery TSP, that is a TSP where different commodities have to be transported from their origin to their destination. The algorithm combines a greedy randomized heuristic and a VND scheme. The local search operators are: a MILP model that solves a restricted version of the problem obtained by fixing the value of a subset of variables, and a $k$-opt operator. This algorithm is an evolution of the approach proposed by the same authors in Rodríguez-Martín and Salazar-González (2011). A further example of a MILP model used as a move operator is the work by Prescott-Gagnon et al. (2009), where an ALNS algorithm is proposed for the solution of the VRPTW where the reconstruction phase is performed through branch-and-price. In particular, branchand-price is used to determine the best place where to insert customers that have been removed during the destruction phase. 


\section{Remarks}

Improvement heuristics are based on the idea of using MILP models in order to modify, and hopefully improve, an existing solution. The nature of the approach, i.e., a oneshot approach or an approach where a MILP model is used for local optimization, highly influence the complexity of the MILP models that are embedded in the solution algorithm. In fact, while in one-shot approaches the MILP models are solved only once and thus a reasonable amount of time can be dedicated to their solution, in approaches belonging to the second class MILP models are solved repeatedly and thus they must give good solutions in a short computing time. The success of these approaches is due to the fact that usually MILP models perform a more exhaustive search than classical heuristic operators. Thus, they are typically used as intensification procedures.

\section{Branch-and-price/column generation-based approaches}

Branch-and-price/column generation algorithms are usually adopted to solve set partitioning formulations. Branch-and-price algorithms have been proved to be successful for the exact solution of a wide variety of routing problems, including some of the most famous and classical ones, like the VRP and VRPTW, and are at the moment the exact leading methodology.

While the branch-and-price scheme is a successful exact method and column generation is a building block of it, their use has been extended to obtain high performing and efficient heuristic algorithms. We call these heuristic approaches branch-andprice/column generation-based approaches. They have the common characteristic of using branch-and-price and/or column generation to build heuristic solutions. However, numerous schemes have been proposed in the literature which differ in terms of how columns are generated and/or of how they are used to obtain a feasible solution.

In the following, we classify the branch-and-price/column generation-based approaches on the basis of the kind of approach proposed. We have identified the following classes: restricted master heuristics, heuristic branching approaches and relaxation-based approaches.

\section{Restricted master heuristics}

One of the most used schemes in the class of branch-and-price/column generationbased approaches is called restricted master heuristic. This scheme is typically embedded in a branch-and-price approach where the set partitioning formulation is solved on a subset of columns generated by the solution of the pricing problem, thus obtaining a feasible solution quickly. The restricted master heuristic is widely used in branchand-price approaches as it enables a quick improvement of bounds and thus a speed up of the exact solution procedure.

An example of restricted master heuristic applied to the solution of the VRPTW is given in Danna and Pape (2005). The authors present a general scheme of cooperation between branch-and-price and local search to help the branch-and-price algorithm finding good solutions at an early stage and test this scheme on the VRPTW. The 
scheme incorporates a restricted master heuristic and a local search applied to solve the master problem which takes the best integer solution found so far as initial solution. In Archetti et al. (2011), a restricted master heuristic is embedded in an exact branchand-price algorithm for the optimal solution of the SDVRP. In order to obtain solutions in a reasonable computing time, the restricted master heuristic is run on a subset of generated columns. The subset of columns is chosen in such a way to guarantee the covering of all customers. The columns determine the sequence of visits of the customers in each route while the quantities are determined in the master problem. The same idea has been applied to different routing problems with profits (RPP). All the following papers propose a branch-and-price exact algorithm embedding a restricted master heuristic. In Archetti et al. (2013b), the capacitated TOP (CTOP) is studied which is a generalization of the TOP where demands are associated with customers, and vehicles are capacitated. The CTOP with incomplete service (CTOPIS) is studied in Archetti et al. (2013a). Finally, in Archetti et al. (2014a, b) the CTOP and the CTOP-IS with split deliveries are analyzed, respectively.

In all works described above, the restricted master heuristic is embedded in an exact branch-and-price algorithm. The pricing problem is solved to optimality and only a subset of columns is used in the set partitioning formulation solved in the restricted master heuristic. On the contrary, the approaches we describe in the following are based on the idea of using heuristic algorithms to generate the columns. The column generation phase may be performed in two different ways: either a heuristic is used which does not take into account the dual information given by the solution of the restricted master problem, or the column generation algorithm is based on the dual information, but only a restricted set of columns is generated. Most of the approaches belong to the first class. This is due to the fact that these approaches are much easier to implement as they simply require a heuristic scheme to generate columns and the set partitioning model. We first analyze the approaches based on heuristic column generation and then describe those based on the use of the dual information provided by the master problem.

One of the first matheuristics for the solution of the VRP is proposed in Foster and Ryan (1976). The authors first present a set partitioning formulation for the VRP and then propose a matheuristic where, in the first phase, a set of petal routes is generated, and then the set partitioning formulation is solved on this set of routes. Petal routes are routes obtained with a heuristic construction method called farthest away cheapest insertion method. They are called petal routes as they resemble petals rooted at the depot. The same heuristic scheme has been improved in Cullen et al. (1981), Renaud et al. (1996) and Ryan et al. (1993). A similar algorithm is proposed in Rochat and Taillard (1995). In the first phase, the VRP is solved through a heuristic which is based on a local search algorithm with probabilistic diversification and intensification strategies. During this phase, all the routes generated by the algorithm are stored in a set $T$. In the second phase, a set partitioning model is solved to optimality to choose the best routes in the set $T$. In Kelly and Xu (1999) the authors propose a two-phase solution approach which resembles the one proposed in Foster and Ryan (1976), where simple and fast construction heuristics are used in the first phase to generate different solutions. In the second phase, a set partitioning model is solved to select the best routes among the set of all routes generated in the first phase. 
Aghezzaf et al. (2006) study the cyclic IRP, that is a problem where a cyclic plan is sought to be operated over a long time horizon. A route is generated for each vehicle which is a sequence of trips, where a trip is a tour starting and ending at the supplier location. These routes are then repeated periodically. The authors propose a formulation where binary variables are used to represent vehicle routes. A column generation approach is proposed where a heuristic algorithm is used to generate columns. This algorithm is an extension of the savings-based heuristic for the VRP. An extension of this approach is applied in Raa and Aghezzaf $(2008,2009)$ to a generalization of this problem.

In Archetti et al. (2008), an optimization-based heuristic is proposed for the solution of the SDVRP. The problem is firstly solved through a TS algorithm. Then, on the basis of the information collected from the solutions explored by the TS, a number of arcs are discarded from the original graph obtaining a reduced graph. A large number of routes are generated on the reduced graph and, finally, a set covering formulation is iteratively solved to select the best routes.

More recently, Schmid et al. (2009) study a VRP with various side constraints encountered in the delivery of ready-mixed concrete. The solution approach incorporates the solution of a multi-commodity network flow problem in a VNS scheme. The VNS is used to generate columns that are then passed to the multi-commodity network flow problem which is solved to optimality. A similar approach is proposed in Parragh et al. (2012) for the heterogeneous dial-a-ride problem with driver constraints.

A general framework based on the generation of routes through a heuristic algorithm and the solution of a set partitioning model is proposed in Subramanian et al. (2013) for the solution of different variants of the VRP. The routes are generated through an iterated local search combined with a VND with random neighborhood ordering (RVND). The routes visited in the solutions that correspond to local optima found by the RVND are stored in a pool which is then passed to the set partitioning model to choose the best ones. The set partitioning model is solved each time a new incumbent solution is found. Mendoza and Villegas (2013) study the VRP with stochastic demands and propose a heuristic solution approach which follows the same idea proposed in Subramanian et al. (2013): in a first phase, routes are generated through a TSP procedure and a splitting procedure, while a set partitioning formulation is solved in the second phase.

In Pillac et al. (2013), the problem of determining the routes for a set of technicians who have to serve a number of tasks is addressed. Each technician has a set of skills, tools, and spare parts while each task has an associated time window and requires specific technician attributes. The authors propose a matheuristic where an initial solution is constructed through a regret constructive heuristic and then improved through an ALNS. All routes generated through the ALNS are used to solve a set covering formulation which is solved to optimality to determine the best set of routes. A similar approach is proposed in Villegas et al. (2013) for the solution of the VRP with trailers.

In Parragh and Schmid (2013), the dial-a-ride problem is solved through a matheuristic where routes are generated through a VNS and then passed to a set covering formulation. The VNS is iteratively run to find negative reduced cost columns. Every time a given number of iterations have elapsed, an LNS is run to improve the best solution found so far. 
Cacchiani et al. (2014) study the periodic VRP (PVRP) which is the problem of finding a set of minimum cost routes for each day of a given planning horizon such that each customer is visited a required number of times, receive every time the required quantity of product, and the number of routes per day does not exceed the total number of available vehicles. The authors propose a set covering formulation and generate columns through an iterated local search algorithm.

An approach where columns are generated on the basis of the dual values provided by the optimal solution of the relaxation of the master problem is proposed in Cohn and Barnhart (2003), where the authors analyze the problem of integrating maintenance routing with crew scheduling in the airline industry. They present an extended crew pairing model which is solved through column generation. A heuristic is proposed where only a subset of columns related to the maintenance routing are generated. The approach is different from those mentioned above in that the column generation phase is performed through a pricing algorithm which uses dual information provided by the solution of the relaxation of the master problem. The difference with respect to an exact branch-and-price algorithm is that only a subset of columns is generated.

\section{Heuristic branching approaches}

Heuristic branching approaches are branch-and-price algorithms where, in order to speed up the convergence of the solution method, branching is performed heuristically with the aim of pruning a large number of nodes of the branch-and-bound tree and thus obtaining a good solution quickly.

The only approach found in the literature belonging to this class is proposed in Cordeau et al. (2001) for the solution of the aircraft routing and crew scheduling problem (ARCSP). The ARCSP consists in determining a minimum-cost set of aircraft routes and crew pairings such that each flight leg is covered by one aircraft and one crew, and side constraints are satisfied. Constraints involve maximum flight time and maintenance requirements. In Cordeau et al. (2001), a Benders decomposition approach is proposed for the solution of the problem which iterates between a master problem that solves the aircraft routing problem and a subproblem that solves the crew pairing problem. Both subproblems are solved through column generation. A heuristic branch-and-bound rule is used to prune a large number of branch-and-bound nodes and obtain integer solutions quickly.

\section{Relaxation-based approaches}

The relaxation-based approaches are characterized by the fact that a feasible solution to the problem is generated from the information provided by the optimal solution of a relaxation of the master problem. Column generation is used to solve the relaxation. Once the relaxed solution is obtained, a heuristic procedure is used to generate a feasible solution to the problem.

This approach is applied to solve the SDVRP in Sierksma and Tijssen (1998) where a set covering formulation is presented with columns corresponding to feasible routes. A relaxed solution is obtained through column generation and the fractional solution 
is then rounded in order to obtain a feasible solution. A similar approach is proposed in Sherali et al. (1999) where a real-case problem arising in maritime logistics is studied. Customer demands can be split and a number of side constraints are added to the basic SDVRP formulation. Two formulations are proposed and solved through column generation. Their relaxation is used to obtain feasible solutions through an ad hoc routing procedure which simulates the solution obtained through the relaxation. In Jin et al. (2008) a set covering formulation for the SDVRP is proposed. The subproblem generates feasible routes and determines how much has to be delivered to each customer in each route. The relaxation of the problem formulation is solved through column generation. Then, a feasible solution is obtained by successively fixing the value of subsets of variables.

\section{Remarks}

Branch-and-price/column generation-based matheuristics are becoming more and more popular. This is due to the success of branch-and-price algorithms developed for the exact solution of routing problems. The scientific community has developed a deep knowledge of column generation approaches, and this knowledge is nowadays transferred also to the development of heuristic schemes. A further advantage of branch-and-price/column generation-based approaches is that they are flexible and easily adaptable to different problem characteristics. Finally, as witnessed from the variety of approaches surveyed in this section, there are different ways of taking advantage of a basic branch-and-price scheme in a heuristic framework. Most of the algorithms adopt the idea of using a set partitioning formulation and rely on heuristic schemes for the generation of columns.

\section{Summary and conclusions}

Table 1 gives a summary of the literature we have surveyed. We have classified the papers on the basis of the kind of approach used and the problem studied. Concerning the problems studied, we have grouped them in the following classes: VRP, SDVRP, pickup and delivery vehicle routing problems (PDVRP), IRP, PRP, LRP, RPP, routing and scheduling problems (RSP).

The following observations can be made:

- The largest number of matheuristics we surveyed were proposed for the most classical class of VRP. Several of these approaches were presented before matheuristics were introduced as a framework.

- The recent literature is focused mainly on improvement heuristics and branch-andprice/column generation-based approaches. In both cases there is a large number of contributions covering a wide variety of problems. This proves that these schemes are efficient and can be easily adapted to different problems.

- Concerning the decomposition approaches, they are still popular in complex problems which involve different kinds of decisions, like IRP, PRP and LRP. This is due to the fact that it is natural and simple to decompose such problems in their 
Table 1 Classification of the literature

\begin{tabular}{|c|c|c|c|}
\hline & Decomposition approaches & Improvement heuristics & $\begin{array}{l}\text { Branch-and-price/column } \\
\text { generation-based } \\
\text { approaches }\end{array}$ \\
\hline \multirow[t]{13}{*}{ VRP } & Fisher and Jaikumar (1981) & Xu and Kelly (1996) & Foster and Ryan (1976) \\
\hline & Koskosidis et al. (1992) & Franceschi et al. (2006) & Cullen et al. (1981) \\
\hline & $\begin{array}{l}\text { Bramel and Simchi-Levi } \\
\text { (1995) }\end{array}$ & Prescott-Gagnon et al. (2009) & Ryan et al. (1993) \\
\hline & $\begin{array}{l}\text { Bramel and Simchi-Levi } \\
\text { (1996) }\end{array}$ & Salari et al. (2010) & Rochat and Taillard (1995) \\
\hline & Flisberga et al. (2009) & Rei et al. (2010) & Renaud et al. (1996) \\
\hline & Demir et al. (2012) & Ngueveu et al. (2010) & Kelly and Xu (1999) \\
\hline & Walteros et al. (2014) & Schmid et al. (2010) & Danna and Pape (2005) \\
\hline & & Yildirim and Çatay (2014) & Schmid et al. (2009) \\
\hline & & & Parragh et al. (2012) \\
\hline & & & Subramanian et al. (2013) \\
\hline & & & Mendoza and Villegas (2013) \\
\hline & & & Villegas et al. (2013) \\
\hline & & & Cacchiani et al. (2014) \\
\hline \multirow[t]{5}{*}{ SDVRP } & & Chen et al. (2007) & Sierksma and Tijssen (1998) \\
\hline & & Gulczynski et al. (2010) & Sherali et al. (1999) \\
\hline & & & Jin et al. (2008) \\
\hline & & & Archetti et al. (2008) \\
\hline & & & Archetti et al. (2011) \\
\hline \multirow[t]{3}{*}{ PDVRP } & & $\begin{array}{l}\text { Rodríguez-Martín and } \\
\text { Salazar-González (2011) }\end{array}$ & Parragh and Schmid (2013) \\
\hline & & $\begin{array}{l}\text { Rodríguez-Martín and } \\
\text { Salazar-González (2012) }\end{array}$ & \\
\hline & & $\begin{array}{l}\text { Wolfler Calvo and } \\
\text { Touati-Moungla (2011) }\end{array}$ & \\
\hline \multirow[t]{2}{*}{ IRP } & Federgruen and Zipkin (1984) & Savelsbergh and Song (2008) & Aghezzaf et al. (2006) \\
\hline & $\begin{array}{l}\text { Campbell and Savelsbergh } \\
\text { (2004) }\end{array}$ & Archetti et al. (2012) & Raa and Aghezzaf (2008) \\
\hline
\end{tabular}


Table 1 continued

\begin{tabular}{|c|c|c|c|}
\hline & Decomposition approaches & Improvement heuristics & $\begin{array}{l}\text { Branch-and-price/column } \\
\text { generation-based } \\
\text { approaches }\end{array}$ \\
\hline & Yu et al. (2008) & Stalhåne et al. (2012) & Raa and Aghezzaf (2009) \\
\hline & Rakke et al. (2011) & Archetti et al. (2013d) & \\
\hline & Coelho et al. (2011) & Song and Furman (2013) & \\
\hline & Coelho et al. (2012) & Maraš et al. (2013) & \\
\hline & Guerrero et al. (2013) & & \\
\hline & $\begin{array}{l}\text { Halvorsen-Weare and } \\
\text { Fagerholt (2013) }\end{array}$ & & \\
\hline & Agra et al. (2014) & & \\
\hline PRP & Chandra and Fisher (1994) & & \\
\hline & Adulyasak et al. (2012) & & \\
\hline & Absi et al. (2014) & & \\
\hline LRP & Yi and Özdamar (2007) & & \\
\hline & Prins et al. (2007) & & \\
\hline & Schittekat et al. (2013) & & \\
\hline & Hemmelmayr et al. (2014) & & \\
\hline RPP & & Archetti et al. (2014c) & Archetti et al. (2013b) \\
\hline & & Archetti et al. (2013c) & Archetti et al. (2013a) \\
\hline & & & Archetti et al. (2014b) \\
\hline & & & Archetti et al. (2014a) \\
\hline RSP & Goel and Meisel (2013) & & Cordeau et al. (2001) \\
\hline & Allaoua et al. (2013) & & Cohn and Barnhart (2003) \\
\hline & & & Pillac et al. (2013) \\
\hline
\end{tabular}

decision components. Another reason is that these approaches proved to behave well for such problems when compared to other heuristic algorithms.

Figure 1 reports the number of papers devoted to matheristics for routing problems per year. We can notice that contributions which date before 2000 are available, but the interest has grown remarkably in the last years. 


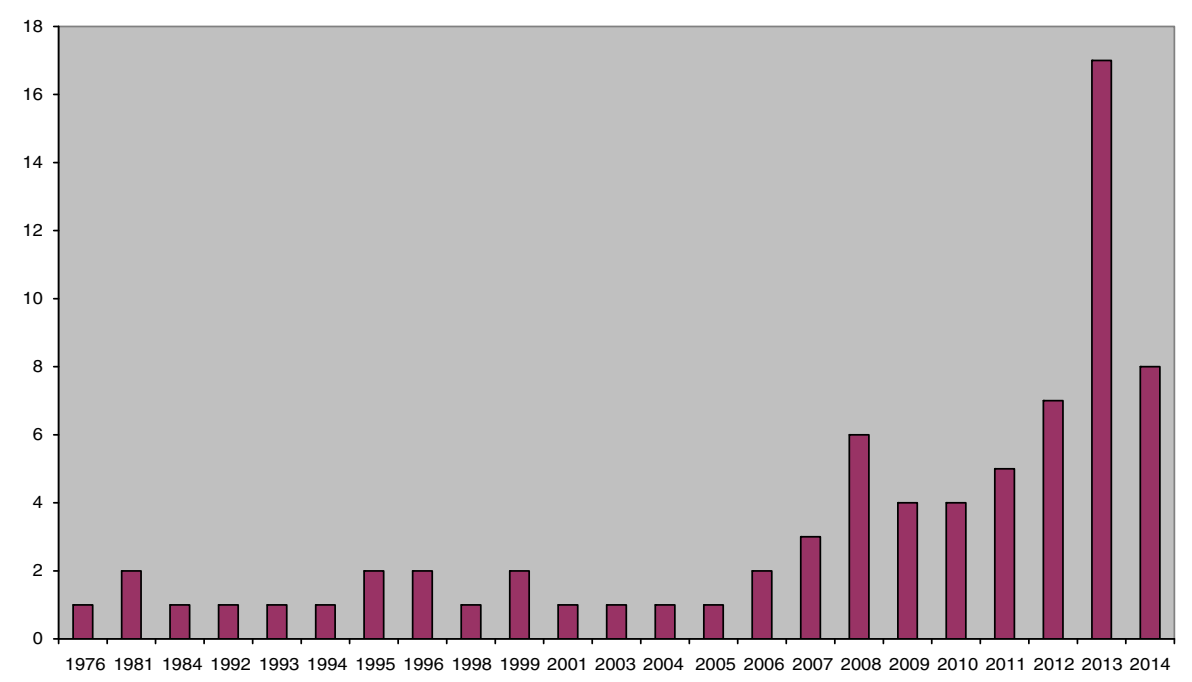

Fig. 1 Number of contributions per year

For a long time, the design of exact and heuristic algorithms have proceeded in parallel, with little interaction between the scientific communities involved in advancing research in the two areas. Exact algorithms are almost always based on a mathematical programming formulation of the problem, whereas heuristics traditionally do not take advantage of it. Commercial and open source software has become available for the solution of MILP models that is powerful, robust, easy to use and simple to interact with. The availability of such software has changed the situation.

Matheuristics make use of mathematical programming formulations and at the same time exploit the specific structure of a problem, keeping the goal of solving large size instances close to optimality. We expect matheuristics will become more and more popular and successful because they can take advantage of state of the art software for the solution of MILP models. Moreover, matheuristics may also become more flexible and robust than traditional heuristics or metaheuristics because, being based on mathematical programming formulations, they may become more easily adaptable to a variety of problems. Moreover, they may request a smaller implementation effort than metaheuristic schemes.

Finally, MILP solvers have become more and more user friendly in the last years. While their use required good programming skills some years ago, nowadays they provide libraries which enable an easy interaction with the solver and inclusion in user codes. What is still missing in the MILP solvers is a tool that interacts with the solver in order to provide information collected from outside and that interacts with, and possibly speed up, the solution process. For example, information provided by heuristic solutions might be used to guide the MILP solver. Some software already include templates that enable users to provide a heuristic solution to the solver which is then used to update bounds. Possibly, this information could be used to more intensively guide the solution process. 
Acknowledgments We acknowledge the contribution of David Pisinger who read a former version of this paper and helped us improve it. We also wish to thank two anonymous referees who provided useful suggestions while reviewing the first submitted version of the paper.

\section{References}

Absi N, Archetti C, Dauzère-Pérès S, Feillet D (2014) A two-phase iterative heuristic approach for the production routing problem. Transp Sci (to appear)

Adulyasak Y, Cordeau J-F, Jans R (2012) Optimization-based adaptive large neighborhood search for the production routing problem. Trasnp Sci. doi:10.1287/trsc. 1120.0443

Aghezzaf E-H, Raa B, Landeghem HV (2006) Modeling inventory routing problems in supply chains of high consumption products. Eur J Oper Res 169:1048-1063

Agra A, Christiansen M, Delgado A, Simonetti L (2014) Hybrid heuristics for a short sea inventory routing problem. Eur J Oper Res 236:924-935

Allaoua H, Borne S, Létocart L (2013) A matheuristic approach for solving a home health care problem. Electron Notes Discret Math 41:471-478

Archetti C, Bertazzi L, Hertz A, Speranza MG (2012) A hybrid heuristic for an inventory-routing problem. INFORMS J Comput 24:101-116

Archetti C, Bianchessi N, Hertz A, Speranza MG (2014a) Incomplete service and split deliveries in a routing problem with profits. Networks 63:135-145

Archetti C, Bianchessi N, Hertz A, Speranza MG (2014b) The split delivery capacitated team orienteering problem. Networks 63:16-33

Archetti C, Bianchessi N, Speranza MG (2011) A column generation approach for the split delivery vehicle routing problem. Networks 58:241-254

Archetti C, Bianchessi N, Speranza MG (2013a) The capacitated team orienteering problem with incomplete service. Optim Lett 7:1405-1417

Archetti C, Bianchessi N, Speranza MG (2013b) Optimal solutions for routing problems with profits. Discret Appl Math 161:547-557

Archetti C, Corberán A, Plana I, Sanchis JM, Speranza MG (2013c) A matheuristic for the team orienteering arc routing problem. In: Proceedings of technical report WPDEM 2013/9, Department of Economics and Management, University of Brescia, Italy

Archetti C, Doerner K, Tricoire F (2013d) A heuristic algorithm for the free newspaper delivery problem. Eur J Oper Res 230:245-257

Archetti C, Guastaroba G, Speranza MG (2014c) An ILP-refined tabu search for the directed profitable rural postman problem. Discret Appl Math 163:3-16

Archetti C, Savelsbergh MWP, Speranza MG (2008) An optimization-based heuristic for the split delivery vehicle routing problem. Transp Sci 42:22-31

Archetti C, Speranza MG (2008) The split delivery vehicle routing problem: a survey. In: Golden B, Raghavan R, Wasil E (eds) The vehicle routing problem latest advances and new challenges, operations research/computer science interfaces series, vol 43. Springer, New York, pp 103-122

Archetti C, Speranza MG (2012) Vehicle routing problems with split deliveries. Int Trans Oper Res 39:3-22

Ball M (2011) Heuristics based on mathematical programming. Surv Oper Res Manag Sci 16:21-38

Bertazzi L, Speranza MG (2012) Matheuristics for inventory routing problems. In: Montoya-Torres JR, Juan AA, Huatuco LH, Faulin J, Rodriguez-Verjan GL (eds) Hybrid algorithms for service, computing and manufacturing systems: routing, scheduling and availability solutions, IGI Global, Hershey, pp 1-14

Bertazzi L, Speranza MG, Savelsbergh MWP (2008) Inventory routing. In: Golden B, Raghavan R, Wasil $\mathrm{E}$ (eds) The vehicle routing problem latest advances and new challenges, operations research/computer science interfaces series, vol 43. Springer, New York, pp 49-72

Boschetti M, Maniezzo V, Roffilli M, Röhler AB (2010) Matheuristics: optimization, simulation and control. In: Blesa M, Blum C, Raidl G, Roli A, Sampels M (eds) Hybrid metaheuristics, lecture notes in computer science, vol 6373. Springer, Berlin, pp 171-177

Bramel J, Simchi-Levi D (1995) A location based heuristic for general routing problems. Oper Res 43:649_ 660

Bramel J, Simchi-Levi D (1996) Probabilistic analysis and practical algorithms for the vehicle routing problem with time windows. Oper Res 44:501-509 
Cacchiani V, Hemmelmayr VC, Tricoire F (2014) A set-covering based heuristic algorithm for the periodic vehicle routing problem. Discret Appl Math 163:53-64

Campbell AM, Clarke L, Kleywegt A (1998) The inventory routing problem. In: Crainic TG, Laporte G (eds) Fleet management and logistics. Springer, Boston, pp 95-113

Campbell AM, Savelsbergh MWP (2004) A decomposition approach for the inventory-routing problem. Transp Sci 38:488-502

Chandra P, Fisher ML (1994) Coordination of production and distribution planning. Eur J Oper Res 72:503517

Chen S, Golden B, Wasil E (2007) The split delivery vehicle routing problem: applications, algorithms, test problems, and computational results. Networks 49:318-329

Coelho LC, Cordeau J-F, Laporte G (2011) The inventory-routing problem with transshipment. Comput Oper Res 39:2537-2548

Coelho LC, Cordeau J-F, Laporte G (2012) Consistency in multi-vehicle inventory-routing. Transp Res Part C 24:270-287

Cohn AM, Barnhart C (2003) Improving crew scheduling by incorporating key maintenance routing decisions. Oper Res 51:387-396

Cordeau J-F, Laporte G, Savelsbergh MWP, Vigo D (2009) Vehicle routing. In: Laporte G, Barnhart C (eds) Transportation, handbooks in operations research and management science, vol 14. North-Holland, Amsterdam, pp 367-428

Cordeau J-F, Stojković G, Soumis F, Desrosiers J (2001) Benders decomposition for simultaneous aircraft routing and crew scheduling. Transp Sci 35:375-388

Cullen F, Jarvis J, Ratiff HD (1981) Set partitioning based heuristics for interactive routing. Networks $11: 125-144$

Danna E, Pape C (2005) Branch-and-price heuristics: a case study on the vehicle routing problem with time windows. In: Desaulniers G, Desrosiers J, Solomon MM (eds) Column generation. Springer, New York, pp 99-129

De Franceschi R, Fischetti M, Toth P (2006) A new ILP-based refinement heuristic for vehicle routing problems. Math Programm 105:471-499

Demir E, Bektaş T, Laporte G (2012) An adaptive large neighborhood search heuristic for the pollutionrouting problem. Eur J Oper Res 223:346-359

Doerner K, Schmid V (2010) Matheuristics for rich vehicle routing problems. In: Blesa MJ, Blum C, Raidl G, Roli A, Sampels M (eds) Hybrid metaheuristics, vol 6373., Lecture notes in computer scienceSpringer, Berlin Heidelberg, pp 206-221

Federgruen A, Simchi-Levi D (1995) Analysis of vehicle routing and inventory-routing problems. In: Ball MO, Magnanti TL, Monma CL, Nemhauser GL (eds) Network routing, handbooks in operations research and management science, vol 8. North-Holland, Amsterdam, pp 297-373

Federgruen A, Zipkin P (1984) A combined vehicle routing and inventory allocation problem. Oper Res 32:1019-1037

Fischetti M, Lodi A (2003) Local branching. Math Program 98:23-47

Fisher ML, Jaikumar R (1981) A generalized assignment heuristic for vehicle routing. Networks 11:109-124

Flisberga P, Lidéna B, Rönnqvist M (2009) A hybrid method based on linear programming and tabu search for routing of logging trucks. Comput Oper Res 36:1122-1144

Foster BA, Ryan D (1976) An integer programming approach to the vehicle scheduling problem. Oper Res Q 27:367-384

Goel A, Meisel F (2013) Workforce routing and scheduling for electricity network maintenance with downtime minimization. Eur J Oper Res 231:210-228

Golden B, Raghavan R, Wasil E (2008) The vehicle routing problem: latest advances and new challenges. Operations research/computer science interfaces series, vol 43. Springer, New York

Guerrero WJ, Prodhon C, Velasco N, Amaya CA (2013) Hybrid heuristic for the inventory location-routing problem with deterministic demand. Int J Prod Econ 146:359-370

Gulczynski D, Golden B, Wasil E (2010) The split delivery vehicle routing problem with minimum delivery amounts. Transp Res Part E 46:612-626

Halvorsen-Weare E, Fagerholt K (2013) Routing and scheduling in a liquefied natural gas shipping problem with inventory and berth constraints. Ann Oper Res 1:167-186

Hemmelmayr VC, Doerner KF, Hartl RF, Vigo D (2014) Models and algorithms for the integrated planning of bin allocation and vehicle routing in solid waste management. Transp Sci 48:103-120 
Jin M, Liu K, Eksioglu B (2008) A column generation approach for the split delivery vehicle routing problem. Oper Res Lett 36:265-270

Kelly JP, Xu J (1999) A set-partitioning-based heuristic for the vehicle routing problem. INFORMS J Comput 11:161-172

Koskosidis YA, Powell WB, Solomon MM (1992) An optimization-based heuristic for vehicle routing and scheduling with soft time window constraints. Transp Sci 26:69-85

Li F, Golden B (2005) Very large-scale vehicle routing: new test problems, algorithms, and results. Comput Oper Res 32:1197-1212

Lin S, Kernighan B (1973) An effective heuristic algorithm for the traveling salesman problem. Oper Res 21:498-513

Maraš V, Lazić J, Davidović T, Mladenović N (2013) Routing of barge container ships by mixed-integer programming heuristics. Appl Soft Comput 13:3515-3528

Mendoza JE, Villegas JG (2013) A multi-space sampling heuristic for the vehicle routing problem with stochastic demands. Optim Lett 7:1503-1516

Min H, Jayaraman V, Srivastava R (1998) Combined location-routing problems: a synthesis and future research directions. Eur J Oper Res 108:1-15

Ngueveu S, Prins C, Wolfler Calvo R (2010) A hybrid tabu search for the m-peripatetic vehicle routing problem. In: Maniezzo V, Stützle T, Voß S (eds) Matheuristics, annals of information systems, vol 10. Springer, New York, pp 253-266

Parragh SN, Cordeau JF, Doerner KF, Hartl RF (2012) Models and algorithms for the heterogeneous diala-ride problem with driver-related constraints. OR Spectr 34(3):593-633

Parragh SN, Schmid V (2013) Hybrid column generation and large neighborhood search for the dial-a-ride problem. Comput Oper Res 40:490-497

Pillac V, Guéret C, Medaglia AL (2013) A parallel matheuristic for the technician routing and scheduling problem. Optim Lett 7:1525-1535

Prescott-Gagnon E, Desaulniers G, Rousseau L-M (2009) A branch-and-price-based large neighborhood search algorithm for the vehicle routing problem with time windows. Networks 54:190-204

Prins C, Prodhon C, Ruiz A, Soriano P (2007) Solving the capacitated location-routing problem by a cooperative lagrangean relaxation-granular tabu search heuristic. Transp Sci 41:470-483

Puchinger J, Raidl GR (2005) Combining metaheuristics and exact algorithms in combinatorial optimization: a survey and classification. In Mira J, Álvarez J (eds) Artificial intelligence and knowledge engineering applications: a bioinspired approach, lecture notes in computer science, vol 3562. Springer, Berlin, Heidelberg, pp 41-53

Raa B, Aghezzaf E-H (2008) Designing distribution patterns for long-term inventory routing with constant demand rates. Int J Prod Econ 112:255-263

Raa B, Aghezzaf E-H (2009) A practical solution approach for the cyclic inventory routing problem. Prod Manuf Logist 192:429-441

Rakke JG, Stalhåne M, Moe CR, Christiansen M, Andersson H, Fagerholt K, Norstad I (2011) A rolling horizon heuristic for creating a liquefied natural gas annual delivery program. Transp Res Part C 19:896911

Rei W, Gendreau M, Soriano P (2010) A hybrid monte carlo local branching algorithm for the single vehicle routing problem with stochastic demands. Transp Sci 44:136-146

Renaud J, Boctor FF, Laporte G (1996) An improved petal heuristic for the vehicle routeing problem. J Oper Res Soc 47:329-336

Rochat Y, Taillard E (1995) Probabilistic diversification and intensification in local search for vehicle routing. J Heuristics 1:147-167

Rodríguez-Martín I, Salazar-González JJ (2011) The multi-commodity one-to-one pickup-and-delivery traveling salesman problem: a matheuristic. In: Pahl J, Reiners T, Voß S (eds) Network optimization, lecture notes in computer science, vol 6701. Springer, Berlin, pp 401-405

Rodríguez-Martín I, Salazar-González JJ (2012) A hybrid heuristic approach for the multi-commodity one-to-one pickup-and-delivery traveling salesman problem. J Heuristics 18:849-867

Ryan DM, Hjorring C, Glover F (1993) Extensions of the petal method for vehicle routeing. J Oper Res Soc 44:289-296

Salari M, Toth P, Tramontani A (2010) An ILP improvement procedure for the open vehicle routing problem. Comput Oper Res 37:2106-2120

Savelsbergh M, Song J-H (2008) An optimization algorithm for the inventory routing problem with continuous moves. Comput Oper Res 35:2266-2282 
Schittekat P, Kinable J, Sorensen K, Sevaux M, Spieksma F, Springael J (2013) A metaheuristic for the school bus routing problem with bus stop selection. Eur J Oper Res 229:518-528

Schmid V, Doerner KF, Hartl RF, Salazar-González JJ (2010) Hybridization of very large neighborhood search for ready-mixed concrete delivery problems. Comput Oper Res 37:559-574

Schmid V, Doerner KF, Hartl RF, Savelsbergh MWP, Stoecher W (2009) A hybrid solution approach for ready-mixed concrete delivery. Transp Sci 43:70-85

Sherali HD, Al-Yakoob SM, Hassan MM (1999) Fleet management models and algorithms for an oil-tanker routing and scheduling problem. IIE Trans 31:395-406

Sierksma G, Tijssen GA (1998) Routing helicopters for crew exchanges on off-shore locations. Ann Oper Res 76:261-286

Song J-H, Furman KC (2013) A maritime inventory routing problem: practical approach. Comput Oper Res 40:657-665

Stalhåne M, Rakke JG, Moe CR, Andersson H, Christiansen M, Fagerholt K (2012) A construction and improvement heuristic for a liquefied natural gas inventory routing problem. Comput Ind Eng 61:245-255

Subramanian A, Uchoa E, Ochi LS (2013) A hybrid algorithm for a class of vehicle routing problems. Comput Oper Res 40:2519-2531

Toth P, Vigo D (2002) The vehicle routing problem. SIAM, Philadelphia

Villegas JG, Prins C, Prodhon C, Medaglia AL, Velasco N (2013) A matheuristic for the truck and trailer routing problem. Eur J Oper Res 230:231-244

Walteros JL, Medaglia AL, Riaño G (2014) Hybrid algorithm for route design on bus rapid transit systems. Transp Sci. doi:10.1287/trsc.2013.0478

Wolfler Calvo R, Touati-Moungla N (2011) A matheuristic for the dial-a-ride problem. In: Pahl J, Reiners T, Voß S (eds) Network optimization, lecture notes in computer science, vol 6701. pp 450-463

Xu J, Kelly JP (1996) A network flow-based tabu search heuristic for the vehicle routing problem. Transp Sci 30:379-393

Yi W, Özdamar L (2007) A dynamic logistics coordination model for evacuation and support in disaster response activities. Eur J Oper Res 179:1177-1193

Yildirim UM, Çatay B (2014) A parallel matheuristic for solving the vehicle routing problems. In: de Sousa JF, Rossi R (eds) Computer-based modelling and optimization in transportation, advances in intelligent systems and computing, vol 262. Springer, Berlin Heidelberg, pp 477-489

Yu Y, Chen H, Chu F (2008) A new model and hybrid approach for large scale inventory routing problems. Eur J Oper Res 189:1022-1040 